\title{
Violence et déraison dans L'Obéissance de Suzanne Jacob
}

\begin{abstract}
:
This article is devoted to the theme of violence in the novel S.Jacob, Obedience.

The author in this confusing fiction with its structure upsetting linearity, painted several characters whose behavior borders on insanity.

At the root of his behavior, education of girls based on violence and segregation. From a history of infanticide S.Jacob challenges the accepted social order and placed under the microscope family relationships. Going back through the generations, she explains that social order by the particular history of Quebec and the socio-economic factors that contributed to this story.
\end{abstract}

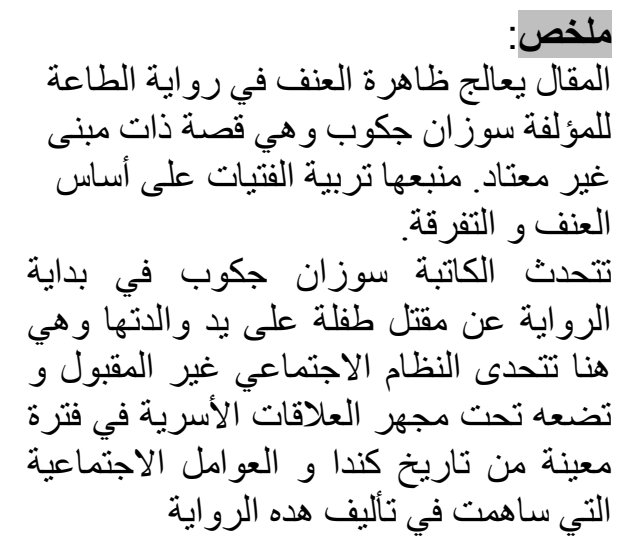

(c) Université des Frères Mentouri Constantine1, Algérie, 2016.

\begin{abstract}
Introduction :
La littérature québécoise a hérité de l'ambivalence qui a prévalue dans son histoire. Faisant partie du continent américain, le Québec a longtemps refusé la domination économique, politique et linguistique anglophone. Au fil du temps, les revendications pour une autonomie se sont succédé jusqu'à la crise sociopolitique de 1970 . La littérature a donc accompagné et souvent mené le projet de société. Placé sous le signe de ces remises en cause et de cette ambivalence, elle ses réfère a la littérature française a laquelle elle ne veut, toutefois, pas lui être subordonné.
\end{abstract}


Les écrivains, en effet, évoquent des thématiques spécifiques au Québec.

Aussi, la littérature québécoise, dans le cadre de ces revendications et sous l'impulsion du féminisme, donne une image particulièrement marquée de la famille. Pour nombre d' écrivains issus de la génération de 1960, « la famille, lieu de socialisation, est liée de près à la perpétuation de l'ordre établi. $\|^{(I)} \mathrm{Et}$ c'est cet ordre établi qui a été au cour de certaines des exigences féministes dont les répercussions ne sont pas achevées à nos jours.

L'obéissance de Suzanne Jacob nous offre un bel exemple de cette représentation de la famille dans la littérature québécoise. Suzanne Jacob est une écrivaine québécoise contemporaine dont l'œuvre a été consacrée par des prix. Ses romans majeurs sont Laura Laur (1983), Prix Paris-Québec et Prix Littéraire du Gouverneur Général, Maude (1988), L'Obéissance (1991), Fugueuses (2005), et Rouge, mère et fils(2001). Elle se situe parmi celles de ces romancières qui se tournent vers les formes avant-gardistes héritées du nouveau roman et des bouleversements de l'écriture introduits à la suite du mouvement féministe.

\section{Synopsis}

Si nous devions présenter de façon succincte le roman de Suzanne Jacob, nous dirions que c'est l'histoire d'un infanticide. Florence Chaillet est accusée d'avoir poussé sa fille, Alice, à se noyer. L'auteure cependant ne se contente pas de proposer ce récit de façon isolée, mais elle l'intègre dans un discours incisif et par des scénarii récurrents de manière à ce que le lecteur ne sorte pas indemne de ce roman. Roman du deuil et de la mort, il laisse une forte impression de malaise.

\section{Une structure complexe}

L'histoire est narrée selon deux récits parallèles, mais qui finiront par se croiser. La structure complexe met en avant ces deux récits de vie qui vont comporter inscrits dans des mises en abymes, contés par des narrateurs différents des récits réflexifs reprenant de façon miniaturisée et parfois bifurquée le sujet développé.

Ce sujet est justement celui des relations qui s'installent au sein des familles. Dès l'incipit, la narratrice, Julie fait un court récit sur une enfant martyrisée, narration qui s'avèrera être une mise en abyme anticipatrice. Puis elle introduit l'un des deux récits parallèles en relatant l'enterrement de Marie, son amie. Dans un troisième temps, un narrateur anonyme narre l'histoire de Florence et Alice, objet du deuxième récit parallèle. C'est alors que Julie, relayée par le narrateur anonyme, reviendra au premier grand récit parallèle, celui sur Marie, pour lui faire croiser le second, puisque Marie s'avère être l'avocate de Florence et qu'elle est morte après avoir défendu Florence accusée du meurtre de sa fille. Au cours de ce deuxième récit parallèle, des personnages-narrateurs 
prennent en charge de nouveaux micro-récits qui ont tous pour thème l'abus de pouvoir de la famille.

\section{Violence et programmes narratifs}

3.1. Récit parallèle sur Florence et Alice, violence de l'éducation.

La violence est présente dans le texte d'abord de manière diffuse, puis elle le devient de façon omniprésente, par la récurrence des actes et des cas.

Premier acte de violence, Florence lors de son mariage gifle son père et en est la première étonnée. De fait toute la rancune accumulée et retenue durant son passif de fillette et de jeune fille s'extériorise dans ce geste incongru d'autant qu'il a pour cadre une cérémonie fortement codifiée. S'il fallait définir ce personnage, ce serait de dire que tout son «vouloir-faire» consiste à s'extraire de l'emprise familiale et sociale. Elle agit de sorte que son mariage lui assure une certaine émancipation.

Ainsi est résumé par les pensées de Florence au cours de cette cérémonie le « vouloir-faire » du personnage, « vouloir-faire » qui constitue l'essentiel de son programme narratif selon la théorie de Greimas ${ }^{(2)}$. Voici comment elle ressent cette étape importante de sa vie, lors de la cérémonie de mariage :

"Lorsqu'elle se tourna vers l'assemblée grouillante des invités, Florence eut le sentiment de n'avoir jamais rencontré ce monsieur et cette dame, les Vésina, ses parents biologiques, ni ce monsieur et cette dame Gouin, ses anciens patrons...ni Gustave Rhéaume gérant du bar l'Exquis qui l'avait engagée à temps partiel comme danseuse nue... » ${ }^{(3)}$

"Le cauchemar de l'enfance finissait. $»^{(4)} \quad$ Mais croyant pouvoir se libérer des pesanteurs et des entraves sociales et familiales grâce à son mariage, Florence a vite fait de déchanter, c'est désormais son époux qui lui imposera ce qu'elle abhorre, la danse. Elle est présentée comme névrosée.

$\mathrm{Au}$ fil du récit l'on découvre le passé de Florence et les raisons de son mal être : Une mère "l'origine de tout» pour laquelle il « était plus facile de gifler sa fille pour n'importe quelle raison que de s'en prendre à son mari ou à ses fils ${ }^{(5)}$

Cherchant à effacer ce passé douloureux fait d'humiliations (elle a travaillé comme bonne chez les Gouin, et pour ces raisons ses camarades la méprisaient, puis comme danseuse et elle en garde un souvenir amer), elle reporte toute son attention sur ses enfants, Rémi et Alice. Un amour possessif, maladif, qui s'achèvera par la mort accidentelle de Rémi suivie de celle, tragique, d'Alice.

Suzanne Jacob présente Florence comme une victime de l'ordre établi par la société patriarcale. Néanmoins, Florence, elle-même, sans en être consciente, est imprégnée des valeurs de cette société. A l'égard de sa fille, Alice, elle reproduit le même schéma de l'éducation qu'elle a reçue. Florence adhère de fait aux valeurs ancestrales que sa mère lui a transmises, valeurs fondées sur la 
ségrégation sexuelle et une autorité sans partage. Aussi, dans son esprit, les pères n'ont pas à s'occuper des filles :

"Alice ne sera jamais assez grande pour que je te laisse t'occuper d'elle. Retiens bien ce que je te dis.» ${ }^{(6)}$ dit-elle à son époux.

"Florence avait redouté ce moment où elle devait partager avec une maîtresse d'école l'éducation de ses enfants...Lorsque la pensée l'effleurait qu'une autre femme allait peut-être punir Alice, tout son corps se rebiffait. Elle seule, Florence, avait ce droit $\gg{ }^{(7)}$

La violence qui accompagne cette autorité est décrite dans des scènes où la mère ne perçoit pas l'amour que pourtant lui voue sa fille, Alice, et la soumet à des punitions qui ressemblent à de la torture.

«Florence faisait brusquement irruption dans la chambre d'Alice. Elle trouvait sa fille assise toute droite sur le bord de son lit. Elle s'appuyait au chambranle pour freiner son exaspération. Alice...tentait de consoler Florence en lui demandant pardon. Ses paroles s'enfonçaient dans l'épaisseur aride qui la séparait de sa mère. "Hypocrite, disait Florence, je vais t'apprendre. " Elle traînait Alice sous la douche et lui tenait la tête sous le jet. ${ }^{(8)}$

Le dernier acte de violence de Florence est enfin exécuté lorsqu'elle emmène sa fille à la rivière et lui demande d'y pénétrer, ne supportant pas qu'Alice ait été témoin d'une scène d'intimité. Un tabou avait été transgressé par la fillette, malgré elle. Florence confie à Marie, son avocate: «Alice m'a vue. Alice me voyait. $»^{(9)}$. En somme c'est un regard interdit qui a tué la petite Alice.

La violence de Florence s'explique par les résonnances en elle de son enfance malheureuse, de sa modeste condition, comme par le désenchantement ayant suivi sa nouvelle vie de femme qui croyait obtenir par le mariage respectabilité et émancipation. Ce sont tous les antagonismes et les frustrations vécus par Florence qui la conduisent au meurtre d'Alice. Est-elle réellement devenue folle comme l'affirme Marie dans sa plaidoirie ?

En définitive, Florence échoue dans son programme narratif qui consiste à s'émanciper et s'affirmer comme être responsable, indépendant de l'autorité familiale. Si elle a exclu ses parents comme ses beaux-parents de sa vie au lendemain de son mariage, elle ne se libère pas de l'emprise des valeurs sociales et des codes qu'elle a intériorisés et qui entravent son "pouvoirfaire $»$.

\subsection{Récit parallèle sur Marie, les répercussions de la violence.}

Marie, l'avocate de Florence, a remporté un grand succès après avoir fait acquitter sa cliente. Mais ce procès lui aura été fatal. Il finit par l'emporter. Car ayant été, elle-même, martyrisée par sa mère dans son enfance, elle subit le contrecoup de ce succès qu'elle regrette en pensant à la petite Alice qu'elle aurait pu/du défendre, car " c'était son histoire, cette histoire ! $\rangle^{(10)}$

"Comment est-ce possible que j'en sois arrivée là ? " ${ }^{(11)}$ se demande-t-elle, lors de la crise violente qui s'empare d'elle lorsqu'elle se rend compte de ce 
qu'elle ressent comme une erreur. Car, Marie avait un secret. Celui de sa propre maltraitance. Elle s'était confiée à Julie :

«-Moi, ma mère me tenait la tête dans l'eau tous les samedis pour m'apprendre à ne pas me révolter...

- Mon père frappait les bébés quand ils ne dormaient pas alors qu'ils auraient dû dormir. $\rangle^{(12)}$

Aussi, Marie, avocate brillante, au faîte de sa carrière, femme émancipée, refuse la maternité, pour ne reproduire sa propre enfance. «Elle refusait de mettre au monde un enfant qu'on menacerait de noyer à son insu. $\|^{(13)}$

De fait, elle veut rompre la chaîne des enfants martyrisés. Elle possède le « vouloir- faire » et contrairement à Florence, elle détient aussi le "savoirfaire ». Elle vit en conformité avec ses principes. Elle déclare pensant à Florence :

«Y penser réveille toujours la même humiliation; Nous ne sommes pas obligées, Florence, nous ne sommes pas obligées, personne ne nous oblige à le faire. $\|^{(14)}$

Le terme « humiliation », le verbe « obliger » répété trois fois rassemblent dans un «nous» englobant Florence, Marie et les femmes en général. Ils expriment le jugement et le rejet de Marie à l'égard des valeurs éternelles perpétuant une condition existentielle féminine difficile. Le sujet de l'énonciation marque ainsi sa position à l'égard des valeurs sociales pesant sur la destinée des femmes de la société du roman.

Mais le procès de Florence Chaillé vient perturber la réalisation du programme de Marie. Défendant une femme estimée victime de la société, et qu'elle présente comme bouc émissaire, elle réalise trop tard qu'Alice méritait aussi d'être défendue. Elle refait le parcours du procès et vérifie que toutes deux, Florence comme Alice, méritaient d'être défendues face à un ordre social injuste. Cet ordre qui est à l'origine, par exemple, de l'infantilisation d'Aglaé, personnage rêvé par Marie, dans l'un des micro-récits.

Torturée par ce qu'elle estime être une erreur, le procès de Florence Chaillé, Marie appelle à son secours son amie, Julie. Dès lors, Marie, désorientée, perdue dans ses contradictions, par un brusque revirement, interrompt donc son programme narratif qui consistait à refuser la maternité pour éviter aux enfants d'être battus, et décide brusquement d'avoir un enfant. Et c'est à ce moment qu'elle contracte le cancer qui va l'emporter et que Julie explique ainsi :

«Les atrocités étaient restées muselées dans les nerfs de Marie. Il y a des gens qui croient que certaines atrocités peuvent provoquer une tumeur au cerveau. ${ }^{(15)}$

Le programme de Marie a été interrompu par la complexité de l'affaire Chaillé qu'elle impute à la société et aux valeurs sur lesquelles celle-ci est fondée. 


\section{Récits miniaturisés, valeurs et discours critique.}

Marie, comme Alice, mais aussi comme Florence, et comme les enfants des micro-récits a été une enfant maltraitée. Le texte en effet, dans des mises en abymes, mentionne d'autres cas de maltraitances parentales ou familiales. Ce sont les cas de :

-la fille portant des traces de coups dans la mise en abyme anticipatrice, et découverte par Julie jouant dans un bac à sable. L'importance de ce court récit tient en sa situation à l'incipit, c'est lui qui donne le la.

- l'homme rencontré dans le train par Marie, et qui raconte que son enfant est mort par suite des négligences parentales.

-Muriel, la relation de Jean, époux de Marie, qui finit par avouer une enfance malheureuse, expliquant la légèreté de sa conduite, une conduite de femme frivole, donnant l'image d'une femme irrésolue, pleine de contradictions.

Les micro-récits concernent également des adultes telle Aglaé, un personnage au comportement insolite, sadique, que le texte présente comme femme infantilisée par ses parents, puis par son époux, qu'elle soumet sciemment à des formes de tortures morales. Mais surtout, le cas de la grandmère de Jean est édifiant. Adriana Rose Saint-Denis, mère de treize enfants attend son quatorzième enfant que le père désirait par superstition, pour éviter le chiffre treize. Mais au moment de la mise au monde, les choses se passent mal, il faut choisir et sauver soit la mère, soit l'enfant : le père et le médecin optent pour l'enfant, un des fils devenu prêtre, venu pour l'extrême onction, explique à sa mère, qui hurle qu'elle ne veut pas mourir, qu'elle doit pourtant s'y résoudre parce que « la loi le veut».

«C'est donc le fils qui «remet toutes les consciences dans le droit chemin et ordonne la mort de sa mère ${ }^{(16)} \quad$ Ainsi, l'Eglise, la science, la loi, le père tous sont d'accord et ligués contre cette pauvre femme qui ne demande qu'à rester en vie.

Le texte, en parlant de loi, fait de la sorte allusion à un contexte spécifique, celui de la période où les femmes étaient considérées dans leur seule fonction de génitrices, période dite de « la revanche des berceaux ». Le Canada français était menacé de disparition, et il fallait songer à augmenter en nombre la population francophone pour contrer la montée en puissance des anglophones. Nécessité faisant loi, les femmes étaient tenues d'enfanter, certaines avaient jusqu'à vingt et une grossesses, la moyenne était justement de quatorze, et ce, sous l'emprise des directives des institutions qui étaient très fortes, la révolution féministe contribuera à un changement radical des mentalités comme du mode de vie.

En effet, la société patriarcale, qui est représentée dans le texte, confirme la suprématie du chef, qu'il soit chef de famille, ou chef de l'état ainsi que le stipulent les lois établies par les hommes. Julie ne se prive pas de le rappeler lorsqu'elle mentionne des personnages référentiels symbolisant des dictatures 
tels : «Hitler, Marcos, Duvalier, Ceausescu ou Kim Il Song » ${ }^{(17)}$ et auxquels elle identifie les parents de Marie qui sont, par Julie, rendus responsables de la mort prématurée de leur fille, Marie. "On devrait pouvoir faire arrêter ses parents »est une conclusion à laquelle aboutit Julie en page 246.

\section{Julie et son programme narratif}

Julie est motivée par le désir de poursuivre l'action de Marie en tentant un changement dans l'ordre des choses. Les valeurs qu'elle défend sont les mêmes que celles de Marie.

Elle aussi se penche sur l'enfance en détresse, pour honorer la mémoire de son amie Marie, elle est donc dans une logique de /devoir-faire/, un devoir moral.

Pour cela, elle détient le savoir (le secret de Marie) et le /savoir-faire/ qui consiste à dénoncer toute situation d'abus d'autorité sur les enfants, et justement avant l'enterrement de Marie, Julie est placée face à une telle situation.

Le/pouvoir- faire/ lui sera acquis lorsqu'elle décidera de briser le pacte de silence qui la liait à la défunte.

La performance consistera à divulguer le secret de Marie et à signaler aux services de protection de l'enfance la présence de la petite fille jouant dans un bac à sable et portant les marques de sévices corporels.

Son programme narratif ne se limitera pas à cet acte, elle relève la passivité de tous ceux (et ils semblent être la majorité) qui feignent d'ignorer ce qui se passe autour d'eux, ainsi que la soumission de ceux qui subissent tout abus d'autorité.

Pour ce qui est de la sanction, nous pouvons dire que Julie est la seule à avoir mené un programme narratif à son terme.

\section{Fonctions et sens du titre.}

La première fonction d'un titre est de nommer le texte, d'informer sur son contenu.

«L'Obéissance » le terme désigne une action. Il résume ainsi le contenu de la fiction et promet de parler de la docilité, de l'autorité et/ou de la soumission à cette autorité. Constitué d'un substantif et d'un déterminant, l'article est un anaphorique qui actualise ici et maintenant ce que Michel Foucault cité par Henri Mitterand. Appelle le « déjà nommé ...la marque du déjà dit, et qu'il suffit de reconnaître $»^{(18)}$ ainsi l'auteur peut se passer de dire ce qu'elle entend par « obéissance ».

Dans ce « déjà dit », l'obéissance ne peut concerner que des situations où un animé cède à l'impulsion d'un autre animé :

- l'animal à l'homme dans une situation de dressage.

- l'enfant à l'adulte, parent, enseignant dans une situation d'éducation ou d'apprentissage.

- l'individu au chef, ce qui fait appel à la notion de pouvoir. 
La deuxième fonction est pragmatique, dans une visée séductive. Elle stimule la curiosité et attire le lecteur dans les filets de la narration. Le titre propose-t-il un récit d'initiation, un récit d'apprentissage, retraçant l'itinéraire d'un personnage qui, confronté à certains domaines de la vie, évolue vers la maturité ?

De fait, à la lecture on se rend compte que le titre est également thématique. S'il ne désigne pas un roman d'initiation, il narre entre autres fictions, l'histoire d'Alice morte par excès d'obéissance.

L'obéissance est également le motif récurrent qui fait pendant à celui de la violence. Julie a mis le doigt sur un ordre des choses, une vision du monde, en cours généralisée, qui lui permet de passer du cas particulier d'Alice ou de Marie dans la relation parents-enfants aux dictateurs sinistrement célèbres du vingtième siècle, et donc à la notion de pouvoir. Le contrat de lecture a, dès lors, été rempli par l'auteur, il s'agit bien de la soumission des enfants aux adultes et par delà, celle des individus à leurs gouvernants par la violence.

Enfin, dans le texte nous repérons plusieurs occurrences du terme «obéissance » et du verbe «obéir», mais nous relevons également le mot " désobéissance » qui introduit le thème de la réaction contre l'excès ou l'abus d'autorité, réaction prônée par Julie et qui place le roman dans une sphère générique particulière, celle de la critique sociale.

\section{Conclusion}

A partir du thème de la violence dans L'obéissance, nous avons pu parcourir le cheminement de la condition des fillettes et des femmes au Québec, à travers la représentation qu'en donne Suzanne Jacob.

Julie dans ce roman, joue le rôle de l'amie, porte-parole de l'auteur, elle dénonce les dysfonctionnements de cette société du roman, qui, si elle n'est pas la société réelle du Québec en porte certaines traces, car ainsi que l'explique Claude Duchet, l'écrivain met en exergue, une vie sociale qu'il transforme grâce à son imaginaire. Il met en place une socialité du roman lui permettant de rendre compte du discours social ambiant, idéologie, institutions, situation sociale et économique. Nous avons avec ce roman de Suzanne Jacob un bel exemple d'interrogations sur les abus de pouvoir dans les relations familiales qui peuvent, selon Suzanne Jacob, conduire à la violence et à la déraison sans que l'on y prenne garde.

\section{Références}

1Lori Saint-Martin, Au-delà du nom. La question du père. Les Presses de l'université de Montréal, Canada, 2010, p.326.

2Pour mener à bien un programme narratif, il faut selon A.J.Greimas posséder les modalités du faire. Le pouvoir-faire et le savoir-faire sont nécessaires pour réaliser la performance, phase essentielle du programme narratif 
3 S.Jacob,L'obéissance.Ed.Boréal.1993.P. 38

4Op.Cit. p. 42

5Op.Cit.p.48

6 Op.Cit. p.101

$7 L$ 'Obéissance .p. 90-91.

8L'Obéissance.p. 89-90.

9L'Obéissance.p. 180.

10Op.Cit. p. 245.

11Op.Cit .p. 174

12Op.Cit. p.245.

13.Op.Cit. p. 242.

14Op.Cit.p.57.

15Op.Cit. p. 242.

16Op.Cit. p. 236.

17Op.Cit.p. 10à12.

18Henri. Mitterrand, in sociocritique, op.cit, p.94.

\section{BIBILIOGRAPHIE}

JACOB Suzanne, L'obéissance, Montréal, Boréal, 1991.

DUCHET Claude, La sociocritique, Paris, Fernand Nathan, coll. Université, information, formation, 1979.

GREIMAS Algirdas Julien, Sémantique structurale, Paris, Larousse, 1966, rééd. Paris, PUF, coll. « Formes sémiotiques », 1986.

PONT-HUMBERT Catherine, Littérature au Québec, Paris, Nathan-université, 1998. 